\title{
Cloning the Genomic Sequence and Identification of Promoter Regions of Bovine Pyruvate Carboxylase ${ }^{1}$
}

\author{
S. R. Hazelton, ${ }^{\star}$ D. M. Spurlock, † C. A. Bidwell, ${ }^{*}$ and S. S. Donkin ${ }^{\star 2}$ \\ *Department of Animal Sciences, Purdue University, West Lafayette, IN 47907 \\ †Department of Animal Science, lowa State University, Ames 50011-3150
}

\begin{abstract}
Pyruvate carboxylase (PC) catalyzes a pivotal reaction in gluconeogenesis and lipid metabolism in liver. In bovine the PC gene is expressed as six $5^{\prime}$ untranslated region (UTR) mRNA variants. The objectives for this study were to clone and sequence the bovine PC gene, determine the intron and exon organization and identify PC promoter region(s). Oligonucleotide sequences that corresponded to the 5' UTR mRNA variants and coding sequence of bovine PC were used to isolate 2 clones from the RPCI-42 bovine bacterial artificial chromosome (BAC) library. Sequencing data confirmed the presence of regions for the $5^{\prime}$ UTR for bovine PC mRNA. The exon arrangement from $5^{\prime}$ to $3^{\prime}$ is 48 (exon I), 41 (exon II), 178 (exon IIIA and IIIB), and 185 (exon IV) bp. Three promoter regions, P3, P2, and P1, adjacent to exon I, II, and IIIA, respectively, were identified based on computer analysis of sequence data. Putative promoters were cloned into a firefly luciferase vector and transiently transfected into H4IIE rat hepatoma cells. All PC promoters demonstrated luciferase activity comparable with the minimal promoter luciferase vector and higher than the promoterless luciferase vector. In addition, $\mathrm{PC}$ promoter 1 exhibited greater luciferase activity compared with PC promoter 2 or 3 . These data provide information about the arrangement of the 4 bovine PC 5' UTR exons, the identity of the promoter regions for the bovine $\mathrm{PC}$ gene, and indicate differences in relative basal activity of the promoter regions.
\end{abstract}

Key words: pyruvate carboxylase, promoter, untranslated region variant

\section{INTRODUCTION}

Pyruvate carboxylase (PC; EC 6.4.1.1) is a regulatory enzyme in gluconeogenesis that catalyzes the biotin-

\footnotetext{
Received July 24, 2007.

Accepted September 21, 2007.

${ }^{1}$ Supported by National Research Initiative Competitive Grant no. 2001-35206-11265 from the USDA Cooperative State Research, Education, and Extension Service.

${ }^{2}$ Corresponding author: sdonkin@purdue.edu
}

dependent carboxylation of pyruvate to form oxaloacetate. There are 6 transcript variants of bovine PC mRNA, which contain a common coding region, and each variant contains a unique $5^{\prime}$ untranslated region (UTR; Agca et al., 2004). Rat PC expresses five 5' UTR variants that are transcribed from 2 different promoters (Jitrapakdee et al., 1997). Transcriptional regulation of rat PC 5' UTR variants from alternative promoters has been implicated in physiological based changes in PC expression, with transcription from the proximal promoter being linked to processes such as gluconeogenesis and lipogenesis and transcription from the distal promoter being linked to anaplerotic functions (Jitrapakdee et al., 1998). To characterize the genomic mechanisms responsible for transcriptional regulation of bovine PC, it was first necessary to identify the location of promoter and regulatory regions within the $5^{\prime}$ UTR of bovine PC. We hypothesize that multiple promoters are responsible for the unique pattern of bovine 5' PC UTR variants. Our objectives were to clone the genomic DNA containing the bovine PC promoter, to identify the location of functional promoter regions and to determine the position of cis-regulatory elements.

\section{MATERIALS AND METHODS}

\section{Bacterial Artificial Chromosome Library Screening}

Genomic DNA for the PC promoter region was obtained by screening the RPCI-42 Male bovine bacterial artificial chromosome (BAC) genomic DNA library (BACPAC Resource Center, Oakland, CA). Three different $40 \mathrm{bp}$ overlapping oligonucleotides probes (overgos) were designed from the $89 \mathrm{bp}$ ( $5^{\prime}$ - ggagcagcggccgtagaggcggtggegacgacttgcggcg) and $110 \mathrm{bp}\left(5^{\prime}\right.$ - agaggcttaacccagaacgtcacttataaacaggcagcgg) regions of bovine PC 5 ' UTR and the open reading frame (5'-tggagtacaagcceatcaagaaggtcatggtggccaacag; Agca et al., 2004) with the Overgo Maker program (Washington University, St. Louis, MO). Each overgo consisted of $24 \mathrm{bp}$ forward and reverse oligonucleotide primers that overlapped by 8 $\mathrm{bp}$, and a 40-bp double-stranded radiolabeled overgo was produced by a Klenow fill-in reaction. Briefly, 10 
pmol of oligonucleotide primers in a volume of $1.0 \mu \mathrm{L}$ were denatured at $90^{\circ} \mathrm{C}$ for $10 \mathrm{~min}$, and placed on ice for $2 \mathrm{~min}$. The denatured overgos were combined with $0.001 \mathrm{mg}$ of bovine serum albumin, $0.005 \mathrm{mCi}{ }^{32} \mathrm{P}-$ $\mathrm{dCTP}, 5 \mathrm{U}$ of Klenow fragment, and $2 \mu \mathrm{L}$ of OLB solution in a final volume of $10 \mu \mathrm{L} /$ reaction at room temperature (RT). Solution OLB consisted of $1 \mathrm{~mL}$ of $1.25 \mathrm{M}$ Tris-Cl ( $\mathrm{pH}$ 8.0), $0.125 \mathrm{M} \mathrm{MgCl}_{2}$ supplemented with $0.019 M \beta$-mercaptoethanol, $0.38 \mathrm{~m} M$ dTTP, $0.38 \mathrm{~m} M$ dATP, $0.38 \mathrm{~m} M$ dGTP, $2.5 \mathrm{~mL}$ of $2 M$ HEPES, and 1.5 $\mathrm{mL}$ of $3 \mathrm{~m} M$ Tris-Cl ( $\mathrm{pH} 7.4$ ), $0.2 \mathrm{~m} M$ EDTA. The overgo reaction mixture was incubated for $1 \mathrm{~h}$, and free nucleotides were removed from the overgo with Microspin G50 Columns (Amersham Pharmacia Biotech, Piscataway, $\mathrm{NJ})$.

The bovine BAC library, printed on a $22 \times 22 \mathrm{~cm}$ nylon high-density filter, was prehybridized for $1 \mathrm{~h}$ in a solution containing $1 \%$ BSA (fraction V), $1 \mathrm{mM}$ EDTA, $3.5 \%$ SDS, and $0.5 M$ sodium phosphate. The ${ }^{32} \mathrm{P}$-labeled overgos were added to the solution and hybridization continued overnight (approximately $16 \mathrm{~h}$ ). After hybridization, the filters were washed for $30 \mathrm{~min}$ at $60^{\circ} \mathrm{C}$ with $4 \times \mathrm{SSC}(20 \times \mathrm{SSC}=0.3 \mathrm{M}$ sodium citrate, 3.0 $M$ sodium chloride, $\mathrm{pH} 7.0$ ), $1 \%$ SDS, followed by 30 min at $60^{\circ} \mathrm{C}$ with $1.5 \times \mathrm{SSC}, 0.1 \% \mathrm{SDS}$, and $30 \mathrm{~min}$ at $60^{\circ} \mathrm{C}$ with $0.75 \times \mathrm{SSC}, 0.1 \%$ SDS. Filters were wrapped in plastic wrap and exposed to Cyclone Storage Phosphor System (Perkin Elmer, Boston, MA) phosphorimaging screens for $1.5 \mathrm{~h}$. The images obtained were analyzed with OptiQuant Analysis Software (Packard Instruments, Meriden, CT). The BAC library filters used in these studies were probed at one week intervals to allow the ${ }^{32} \mathrm{P}$ signal to subside. Two BAC clones, RPCI$42,243 \mathrm{~A} 6$ and RPCI-42, $147 \mathrm{~K} 3$, were identified that hybridized to all 3 overgos and were purchased from BACPAC Resources Center (Oakland, CA). Both BAC were used for subcloning and sequencing.

\section{BAC DNA Isolation}

Upon receipt the BAC clones were streaked on $\mathrm{LB}$ agar plates containing $20 \mu \mathrm{g} / \mathrm{mL}$ chloramphenicol and grown overnight at $37^{\circ} \mathrm{C}$. Individual colonies were inoculated into $2 \mathrm{~mL}$ of Circlegrow broth (Bio 101, Carlsbad, CA) containing $20 \mu \mathrm{g} / \mathrm{mL}$ of chloramphenicol and grown overnight at $37^{\circ} \mathrm{C}$ with shaking. The BAC DNA was isolated via alkaline lysis miniprep. Briefly, the $E$. coli cells from the overnight culture were pelleted and resuspended in $0.3 \mathrm{~mL}$ of Tris-EDTA (TE; $10 \mathrm{~m} M$ Tris (pH 8.0), $1 \mathrm{~m} M$ EDTA) containing $100 \mu \mathrm{g} / \mathrm{mL}$ RNase A solution. The cells were lysed with $0.3 \mathrm{~mL}$ of $0.2 \mathrm{~N}$ $\mathrm{NaOH}, 1 \%$ SDS followed by addition of $0.3 \mathrm{~mL}$ of $3 M$ potassium acetate, $\mathrm{pH}$ 5.5. The protein and cellular debris were pelleted by centrifugation at $13,800 \times g$ for
$10 \mathrm{~min}$. The supernatant was precipitated with an equal volume of ice-cold isopropanol and centrifuged for $15 \mathrm{~min}$ at $13,800 \times \mathrm{g}$. The DNA pellet was rinsed with $70 \%$ ethanol and centrifuged at $13,800 \times g$ for 5 min, air-dried, and resuspended in $1 \times \mathrm{TE}$ buffer. To assess the purity of the preparation, the DNA was digested with EcoR I, and size separated by electrophoresis through a $0.8 \%$ agarose Tris-acetate EDTA gel.

\section{Southern Blots, Subcloning, and Sequencing}

Fragments of the BAC clones were generated by restriction enzyme digestion with EcoR I, Hind III, Kpn I, BamH I, and Sac I, as well as double digestions with the same enzymes. Restriction enzyme digested BAC were separated through a $0.8 \%$ agarose $1 \times$ TBE (Trisborate-EDTA) gel and transferred to Genescreen membrane (NEN Life Science, Boston, MA) by capillary transfer. The DNA was crosslinked to the membrane using ultraviolet light. The membrane was prehybridized in solution containing $1 \%$ SDS, $2 \times$ SSC, $10 \%$ dextran sulfate, $50 \%$ formamide, $5 \times$ Denhardt's ( $2 \mathrm{~g}$ of Ficoll, $2 \mathrm{~g}$ of polyvinylpyrolidone, $2 \mathrm{~g}$ of BSA per $200 \mathrm{~mL}$ of water) for at least $6 \mathrm{~h}$. Membranes were probed using ${ }^{32} \mathrm{P}$-labeled DNA generated using overgo DNA as indicated above or a PCR product for the 185-bp exon. Probes were combined with $0.5 \mathrm{mg}$ of herring sperm in a volume of $1 \mathrm{~mL}$, denatured for $10 \mathrm{~min}$ at $95^{\circ} \mathrm{C}$, and chilled on ice for $20 \mathrm{~min}$ and hybridized overnight at $42^{\circ} \mathrm{C}$. After hybridization, the membrane was washed once at RT with $2 \times \mathrm{SSC}$ for $10 \mathrm{~min}$, twice with $2 \times \mathrm{SSC}$, $1.0 \% \mathrm{SDS}$ at $42^{\circ} \mathrm{C}$ for $20 \mathrm{~min}$, and twice with $0.2 \times \mathrm{SSC}$, $1.0 \% \mathrm{SDS}$ at $42^{\circ} \mathrm{C}$ for $20 \mathrm{~min}$. After washing, the membrane was wrapped in plastic wrap and exposed to Fuji Super Rx medical x-ray film (Fuji Film, Edison, NJ).

The migration of DNA fragments containing bovine PC 5' UTR DNA determined by Southern blots, described above, were used to locate, isolate BAC DNA fragments for subcloning and sequencing. A second aliquot of BAC DNA digest, was separated by agarose gel electrophoresis, quantified by visual comparison to Biorad Precision molecular mass standards (Hercules, $\mathrm{CA}$ ), and isolated from gel fragments using a QIAquick Gel Extraction Kit (Qiagen, Valencia, CA). The DNA fragments were subcloned into pGEM-3Z (Promega, Madison, WI) and transformed into competent JM109 or DH5 $\alpha$ E coli cells. The transformation reaction was plated on $\mathrm{x}$-Gal/IPTG plates and incubated for $16 \mathrm{~h}$ at $37^{\circ} \mathrm{C}$. Transformed colonies were inoculated into culture tubes that contained $8 \mathrm{~mL}$ of LB broth with 100 $\mu \mathrm{g} / \mathrm{mL}$ of ampicillin and grown overnight at $37^{\circ} \mathrm{C}$ with shaking. The plasmid DNA was isolated with a Wizard Plus SV Miniprep kit (Promega) and quantified. The presence of the bovine $\mathrm{PC}$ genomic sequences was veri- 
fied by Southern blot or PCR. A total of 7 subclones were generated from BAC RPCI-42 243A6 and RPCI42 147K3. One clone contained overlapping sequence for the BAC. Sequence data for these clones has been deposited in GenBank (Accession numbers DQ072585, DQ072584, DQ072583， DQ072582， DQ072580, DQ072581).

An aliquot of each plasmid was sequenced at the Low Throughput Lab of Purdue University using the ABI 3700 sequencer (Amersham Biosciences, Piscataway, NJ). The SP6 and T7 promoter primers for pGEM-3Z were used for the first round of sequencing. The inserts ranged from 2 to $12 \mathrm{~kb}$, so sequencing using the SP6 and $\mathrm{T} 7$ primers did not yield the entire insert sequence. To obtain the complete insert sequence, gene specific sequencing primers were designed (Oligo 5.0, Cambio, Cambridge, UK) from the partial sequence information obtained. A contiguous sequence for bovine PC genomic DNA was generated from these overlapping fragments using the Contig Express feature of Vector NTI (Invitrogen, Frederick, MD).

Pyruvate carboxylase 5' UTR exon size was verified via PCR of bovine genomic DNA samples. Genomic DNA from dairy cow liver samples was isolated with the Wizard Genomic DNA Purification Kit (Promega). Primers that corresponded to the $5^{\prime}$ and $3^{\prime}$ end of the exon in question were used to amplify bovine genomic DNA. The PCR products were separated through a $0.8 \%$ agarose Tris-acetate EDTA gel, and the size of the products was verified by comparison with Biorad Precision molecular mass standards (Hercules, CA).

\section{Sequence Analysis}

The nucleotide-nucleotide Basic Local Alignment Search Tool (BLASTn; Altschul et al., 1990) was used to verify the position of exons and bovine short interspersed nucleotide elements within the 5' UTR of bovine PC. A BLASTn analysis of the high-throughput gene sequence database was used to compare sequence data to existing sequence data for the bovine genome. The combined search query function of the Transcription Element Search System (TESS; University of Pennsylvania, Philadelphia, PA; www.cbil.upenn.edu/tess) was used to identify promoter elements, as well as other known putative transcription factor binding sites of interest, upstream of the $5^{\prime}$ UTR exons and the PC translation start site. Promoter regions were identified by the presence of putative selective transcription factor 1 (Sp1), CAAT binding protein (CBP), or TATA binding protein (TBP) sites. Only sequence sites that yielded a perfect sequence match for transcription factor binding sites of interest were chosen. Criteria for putative transcription factor site selection were also based on stan- dards listed on the TESS Web site, which included a log-likelihood (La) score of 12 or higher, La/length of site score of $2, \mathrm{La} / \mathrm{L} \_\mathrm{M}$ score of 1 ( $\mathrm{L} \_\mathrm{M}$ is the maximum La score for model) and L_M - La score of 0 .

\section{Promoter-Luciferase Constructs}

Functionality of the putative promoter was determined by linking each promoter sequence to a firefly luciferase reporter gene sequence. The promoter-luciferase constructs contained 281, 1,093, and 610 bases relative to the first exon base within promoters $\mathrm{P} 1, \mathrm{P} 2$, and P3, respectively. Regions tested for P1, P2, and P3 corresponded to sequences 612518 through 613127 , 655864 through 656956, and 660337 through 660617 respectively of the Bos taurus chromosome 29 genomic contig, reference assembly (Accession number NW_001494541). Briefly, each promoter region was amplified via PCR, and the PCR product was purified by agarose gel electrophoresis and a QIAquick Gel Extraction Kit gel. The purified PCR product was ligated into the multiple cloning site of pGL3-Basic plasmid (Promega) upstream of the coding region for firefly luciferase. Correct orientation of the inserted promoter region was verified by sequencing or restriction enzyme digestion.

\section{Hepatoma Cell Culture and DNA Transfections}

Rat hepatoma H4IIE cells (ATCC, Manassas, VA) were grown to $80 \%$ confluence in 6 -well plates in complete medium (Dulbecco's Modified Eagle's Medium (DMEM), 10\% fetal bovine serum, 1\% antibiotic, antimycotic solution; (Sigma, St. Louis, MO) and used to test the functionality of bovine PC promoters. The PC promoter-luciferase plasmids described above were transfected into hepatoma cells and expression of luciferase protein was used to assess promoter function. The plasmid pRL-CMV that expresses Renilla luciferase was cotransfected as a normalization control. The plasmid pGL3-Basic (Promega) is a promoterless luciferase plasmid and served as a negative control. The plasmid pGL3-Promoter (Promega) contains a luciferase reporter driven by the SV40 promoter and served as a positive control. Briefly, a volume of $10 \mu \mathrm{L}$ of Lipofectin transfection reagent (Invitrogen, Carlsbad, CA) was gently mixed with $90 \mu \mathrm{L}$ of DMEM and incubated at RT for $45 \mathrm{~min}$. The Lipofectin mixture was added to the plasmid DNA mixtures and incubated for $10 \mathrm{~min}$ at RT. The plasmid DNA mixture contained $1.49 \mu \mathrm{g}$ of PC promoter-luciferase reporter plasmid DNA, pGL3Promoter, or pGL3-Basic and $10 \mathrm{ng}$ of Renilla luciferase plasmid (pRL-CMV, Promega) in DMEM to a total volume of $100 \mu \mathrm{L}$. Complete medium was removed from 
the cells 45 min prior to transfection and replaced with $2 \mathrm{~mL}$ of DMEM. To initiate the transfection, $800 \mu \mathrm{L}$ of DMEM was added to the 200- $\mu \mathrm{L}$ lipofectin and DNA mix, the DMEM was removed from the cells and replaced with the $1 \mathrm{~mL}$ solution. Cells were incubated for $5 \mathrm{~h}$ at $37^{\circ} \mathrm{C}$. After the $5 \mathrm{~h}$ incubation, $2 \mathrm{~mL}$ of complete medium were added to each well, and the incubation was continued for $28 \mathrm{~h}$. All transfections were performed in triplicate.

\section{Luciferase Assay}

After $28 \mathrm{~h}$, the complete media was withdrawn from the cells, and cells were rinsed with $1 \times$ PBS. The PBS was removed, and $250 \mu \mathrm{L}$ of $1 \times$ passive lysis buffer (Promega) was added to each well. Cells were lysed by scraping, and the lysate was transferred to a sterile microcentrifuge tube, centrifuged at $12,000 \times g$ for 1 $\mathrm{min}$, and the cleared lysate analyzed for luciferase activity. Assays were initiated by the addition of $100 \mu \mathrm{L}$ of either luciferase detection reagent (Promega) or Renilla luciferase detection reagent (Promega) and luminescence was assayed using Tecan GENios Pro spectrofluorometer with Magellan 5.0 software (Tecan, Research Triangle Park, NC). Data were normalize for transfection efficiency by dividing the relative light units detected for firefly luciferase by the corresponding Renilla luciferase relative light units value adjusted for background.

\section{Statistical Analysis}

Normalized firefly luciferase values were log transformed and analyzed as a randomized complete block design, using the GLM procedure of SAS (SAS Inst. Inc., Cary, NC). Single degree of freedom contrasts were used to test for differences in luciferase activity between plasmids, and differences between means were considered different when $P<0.01$.

\section{RESULTS}

\section{Bovine Pyruvate Carboxylase 5' UTR Exon Arrangement}

Sequencing information obtained from isolated BAC clones has been deposited with Genbank. These sequences correspond to the $5^{\prime}$ UTR fragments previously identified (Agca et al., 2004) and the regions identified as promoters (GenBank accession no. DQ072580 and DQ072581). In addition sequences adjacent to these regions, ranging from 1.3 to $3.8 \mathrm{~kb}$, have also been deposited (GenBank accession no. DQ072582, DQ072583, DQ072584, and DQ072585).
Regions of genomic DNA that contained each of the UTR fragments identified for bovine (Agca et al., 2004) were isolated and sequenced. A BLAST comparison of our sequence data with the bovine genome draft sequence build 3.1 (GenBank accession no. NW_001494541.1) identified a contiguous sequence within chromosome 29 that corresponded to the bovine PC gene. Model maker (NCBI; http://www.ncbi.nlm.nih.gov; accessed July 23, 2007) revealed that all exons and putative promoter sites are located within a region approximately $80 \mathrm{~kb}$ upstream of the translation start site. The bovine PC gene contains 4 exons that give rise to six different $5^{\prime}$ UTR transcripts (Figure 1). The genomic sequence indicates that UTR sequence data for the 110- and 68-bp units of bovine PC $5^{\prime}$ UTR A, B, $\mathrm{C}$, and $\mathrm{F}$ are present as 178 bp continuous nucleotides. Therefore the exons I, II, IIIA, IIIB, and IV within the bovine pyruvate carboxylase gene correspond to the unique 48-, 41-, 110-, 68-, and 185-bp sequences previously identified by our laboratory (Agca et al., 2004).

\section{Bovine Pyruvate Carboxylase Promoter Region Identification}

Three distinct bovine PC promoter sites were identified based on the criteria described above. The promoter (P1) most proximal to the coding region of bovine pyruvate carboxylase is contained within the region located 200 bp 5' of exon IIIA within the bovine PC gene (GenBank accession no. DQ072580). This promoter contains 5 putative Sp1 sites, but no CBP or TATA sites (Figure 2). The middle promoter (P2) is located within 1,093 bp 5' of the start of the 41-bp exon II and contains one putative TBP and six Sp1 sites (Figure 3; GenBank accession no. DQ072581). The most distal promoter (P3) is located within a region $281 \mathrm{bp} 5^{\prime}$ of exon I and contains one putative CBP and eight Sp1 sites (Figure 4; GenBank accession no. DQ072581). In addition, putative regulatory elements, such as peroxisome proliferator-activated receptor (PPAR), CAAT/enhancer-binding protein alpha $(\mathrm{CEBP} \alpha)$, glucocorticoid response element (GR), and D-site binding protein (DBP), were present within the promoter sequences identified.

\section{Bovine Pyruvate Carboxylase Promoter Activity}

Each of the 3 bovine PC promoters drives expression of luciferase activity in separate promoter-reporter constructs (Figure 5) indicating functionality of these DNA sequences to drive protein expression in liver cells. All 3 PC promoters demonstrated luciferase activity greater than the negative control pGL3-Basic $(P<0.01)$ and comparable to the positive control pGL3-Promoter $(P<$ $0.01)$. In addition the activity of $\mathrm{P} 1$ for bovine $\mathrm{PC}$ was greater than $(P<0.01)$ P2 or P3. 


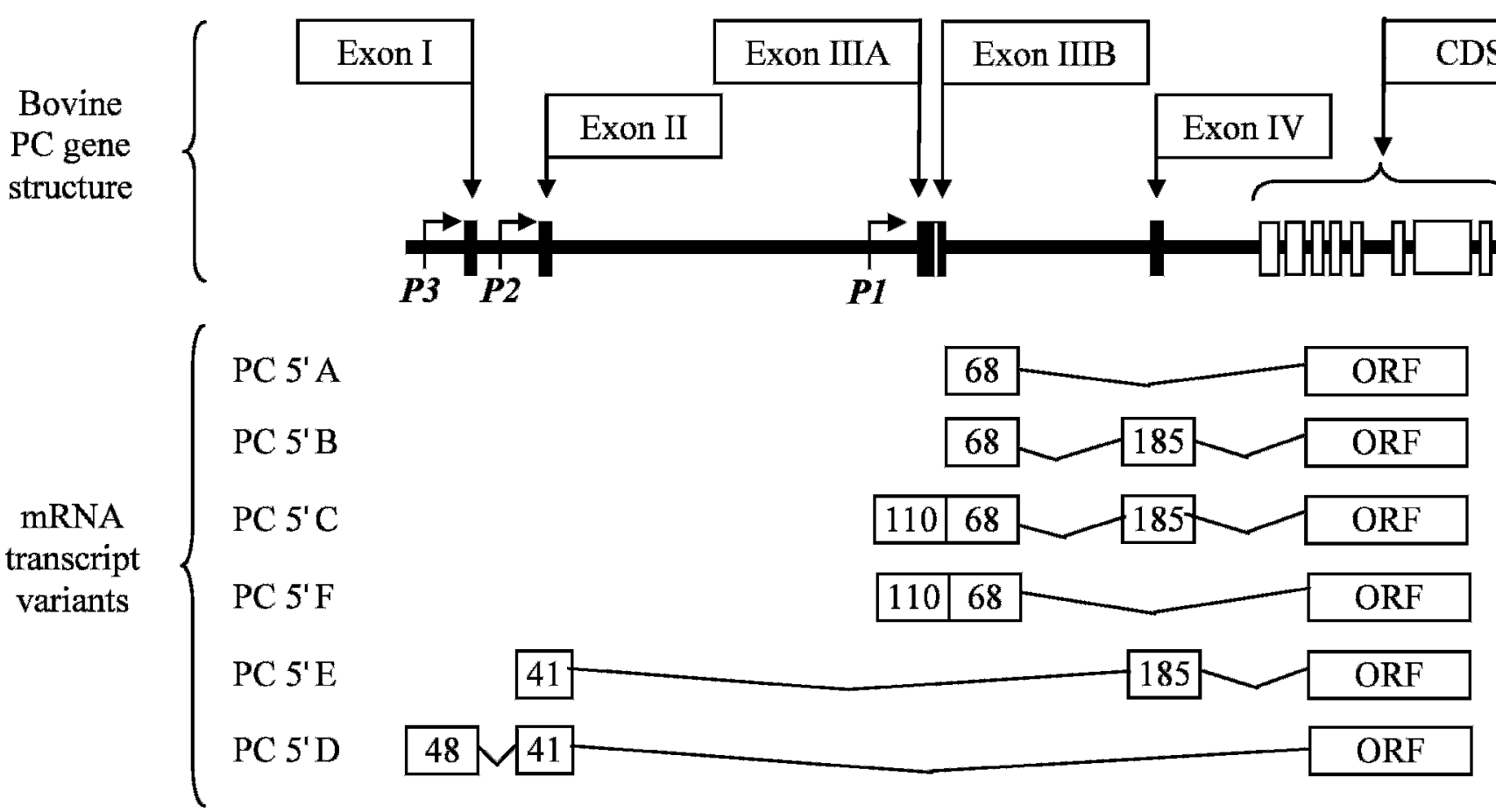

Figure 1. Bovine pyruvate carboxylase (PC) gene arrangement and origin of $5^{\prime}$ UTR mRNA variants. The origin of bovine PC $5^{\prime}$ UTR variants were determined from genomic DNA sequence. The exon arrangement is indicated at the top and the resulting bovine PC 5' UTR transcript variants are indicated to the left. Promoter (P1, P2, P3) locations are indicated relative to exons. Promoter 1 is responsible for transcription of bovine PC 5' UTR variants A, B, C, and F. Promoter 2 generates bovine PC 5' UTR E, and P3 transcribes bovine PC 5' UTR D. The open reading frame $(\mathrm{ORF})$ common to all variants is indicated.

\section{DISCUSSION}

The data suggest that the origins of multiple UTR variants for bovine $\mathrm{PC}$ are the product of three distinct promoters and alternative splicing. The bovine PC gene contains 4 exons and 3 promoters in the region $5^{\prime}$ of the coding region. Transcription from these promoters and the resulting bovine PC 5' UTR variants are consistent with previously described models of exon inclusion, exclusion, and alternative $5^{\prime}$ site splicing mechanisms for eukaryotic genes (Modrek and Lee, 2002). The bovine PC 5' UTR variants A, B, C, D, and F (Agca et al., 2004) are the result of exon inclusion and exclusion splicing, whereas variants A and B result from alternative $5^{\prime}$ transcription start sites within exon III (Figure 1).

Pyruvate carboxylase transcripts for rat, human, and bovine contain multiple 5' UTR, but transcripts within each species share the same open reading frame and thus produce the same protein (Agca et al., 2004; Jitrapakdee et al., 2006). In general, genes with $5^{\prime}$ UTR variants are the product of transcription from multiple promoters, and the $5^{\prime}$ UTR variants display differential transcriptional profiles, as well as different translation efficiencies (Landry et al., 2003). Six PC 5' UTR variants have been identified and characterized for bovine (Agca et al., 2004). The presence of these variants suggests control of synthesis at the transcriptional level that is at least as complex as that described for rat (Jitrapakdee et al., 1996, 1997). Based on the similarity in the nucleotide composition of transcripts, as well as the arrangement of portions of the 5' UTR fragments (Agca et al., 2004), we hypothesized that bovine PC was also transcribed from multiple promoters. Data from the current experiment confirm and extend this hypothesis and serve to identify 3 unique promoter regions within the bovine PC gene.

The proximal promoter of bovine shares similarities with the proximal promoter for other nonruminant mammalian PC genes that have been characterized, namely rat, mouse, and human. It should be noted that the response elements identified for bovine are putative and need to be confirmed by experimental methods. Therefore, discussion of the similarities and differences between nonruminant and bovine $\mathrm{PC}$ genes is tentative. However, in both cases the proximal promoter drives UTR variant expression that is lipogenic and gluconeogenic specific (Agca et al., 2004; Jitrapakdee et al., 2006). In mouse adipocytes, the proximal promoter is positively regulated by PPAR $\gamma 2$ (Jitrapakdee et al., 2005). Analysis of the bovine PC proximal promoter 
Figure 2. Nucleotide sequence and putative transcription factor binding sites for bovine pyruvate carboxylase (PC) promoter P1. Sequence information corresponding to 610 bases immediately $5^{\prime}$ of exon IIIA of the bovine PC gene was analyzed using the Transcription Element Search System. The exon immediately adjacent to the promoter sequence is indicated by the boxed sequences. Putative transcription factor binding sites are indicated as follows: Sp1 = selective transcription factor-1; PPAR = peroxisome proliferator-activated receptor; and Zta $=$ Epstein-Barr virus Z protein.

also reveals the presence of a putative PPAR response element within $200 \mathrm{bp}$ of exon IIIA. Unlike nonruminants, a putative CREB site was not detected in the proximal promoter of bovine PC. Expression of bovine PC mRNA in liver is elevated during fasting (Velez and Donkin, 2005) and the transition to lactation (Greenfield et al., 2000), physiological states that are linked to elevated NEFA concentrations in blood. Therefore, the rise in NEFA in blood and accompanying increased NEFA in liver may lead to activation of PC gene expression via PPAR mediated events. Examination of the profile of $\mathrm{PC}$ variants present at calving reveals bovine PC 5'A is most abundant (Agca and Donkin, 2002), which would be consistent with enhanced activity of the P1 promoter. However, tests of function assays are necessary to determine any specific involvement for the putative PPAR response element in $\mathrm{P} 1$ of bovine $\mathrm{PC}$ in regulating $\mathrm{PC}$ mRNA abundance at calving.

The presence of a TATA box or an initiator (INR) element are key components of a functional promoter (Novina and Roy, 1996; Roeder, 1996). All 3 bovine PC promoters have several Sp1 sites, and P1 and P3 have a pyrimidine-rich site (YYaNt/aYY), indicating possible INR elements. Similar to the proximal promoter of rat PC, the bovine PC promoter P2 contains a TATA box, but also lacks a CAAT box and the housekeeping initia- tor protein sequence (HIP-1) found in the rat PC proximal promoter (Jitrapakdee et al., 1997). Bovine PC promoter P3 has a CAAT box, but no TATA box, and the proximal promoter, $\mathrm{P} 1$, has neither a TATA box nor a CAAT box. We analyzed the proximal rat PC promoter region (Genbank accession no. U81515) using the same conditions in TESS applied to the bovine PC promoter. Rat PC has a putative INR element located in the $3^{\prime}$ region $(1,145$ to $1,151 \mathrm{bp})$, and the distal rat PC promoter (Genbank accession no. U95043) has 2 INR elements in the $3^{\prime}$ region located at bases 1,112 through 1,118 and 1,124 through 1,130 . Therefore a number of similarities exist between that rat and bovine with regard to location of INR elements. In both species, these elements are located within $35 \mathrm{bp} 5^{\prime}$ of the transcription start sites and their nucleotide sequences display a high degree of identity. Conversely, there also appears to be several unique features of the bovine PC gene promoters that warrant further investigation.

Based on computer-assisted analysis, the rat PC and bovine PC promoters display similarity in core promoter elements and both have putative transcription factor binding sites for c-Myb, PPAR, CAC-binding protein, and nuclear factor 1 (NF-1; Jitrapakdee et al., 1997). However, the presence of an additional promoter in bovine PC makes it difficult to directly compare pro- 


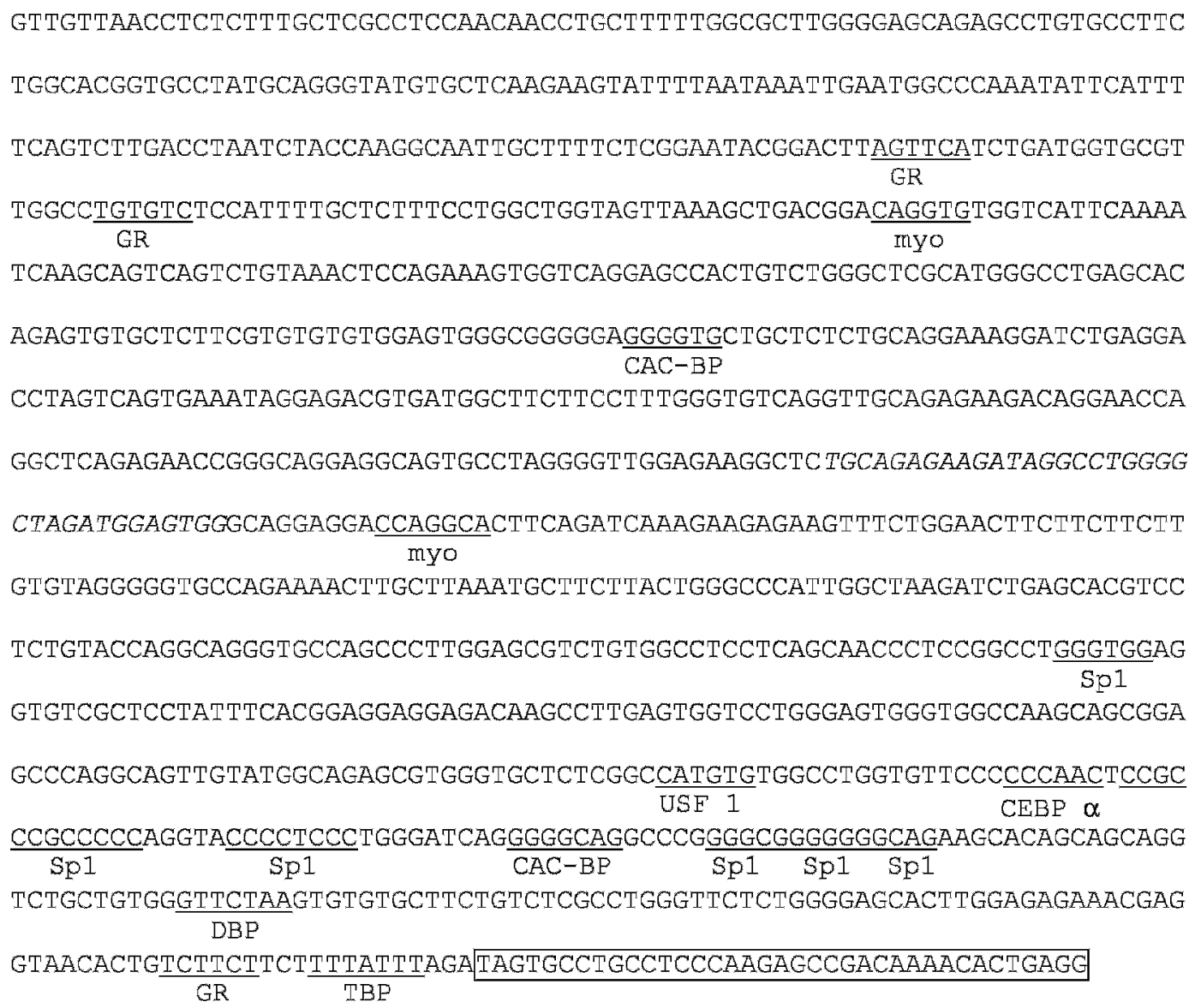

Figure 3. Nucleotide sequence and putative transcription factor binding sites for bovine pyruvate carboxylase (PC) promoter P2. Sequence information corresponding to 610 bases immediately $5^{\prime}$ of exon II of the bovine PC gene was analyzed using the Transcription Element Search System. The exon immediately adjacent to the promoter sequence is indicated by the boxed sequences. The start of the alternative splice site that generates the 68-bp fragment of PC 5' UTR A and B is indicated by the shaded nucleotide within the exon sequence. Putative transcription factor binding sites are indicated as follows: $\mathrm{GR}=$ glucocorticoid receptor; CAC-BP $=\mathrm{CAC}$ binding protein; CEBP $\alpha=\mathrm{CCAAT} /$ enhancer binding protein-alpha; $\mathrm{DBP}=$ albumin D-box binding protein; myo = myogennin; Sp1 = selective transcription factor-1; TBP = TATA-binding protein; and USF = upstream stimulatory factor.

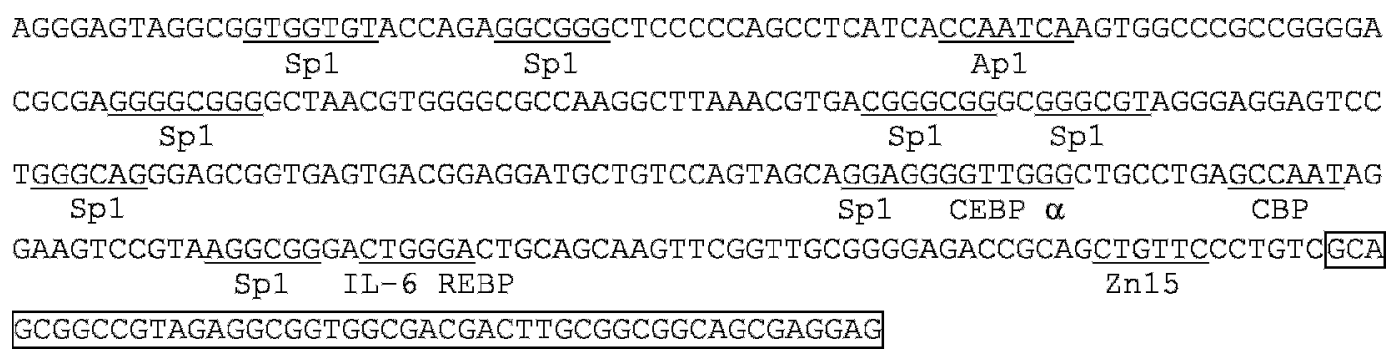

Figure 4. Nucleotide sequence and putative transcription factor binding sites for bovine pyruvate carboxylase (PC) promoter P3. Sequence information corresponding to 610 bases immediately $5^{\prime}$ of exon I of the bovine PC gene was analyzed using the Transcription Element Search System. The exon immediately adjacent to the promoter sequence is indicated by the boxed sequences. Putative transcription factor binding sites are indicated as follows: Ap-1 = activator protein 1; GR = glucocorticoid receptor; CAC-BP $=\mathrm{CAC}$ binding protein; $\mathrm{CEBP} \alpha=$ CCAAT/enhancer binding protein-alpha, CBP = CREB-binding protein; DBP = albumin D-box binding protein; IL-6 REBP = interleukin-6 response element binding protein; myo = myogennin; Sp1 = selective transcription factor-1; TBP = TATA-binding protein; USF $=$ upstream stimulatory factor; and $\mathrm{Zn}-15$ = zinc finger 15 protein. 


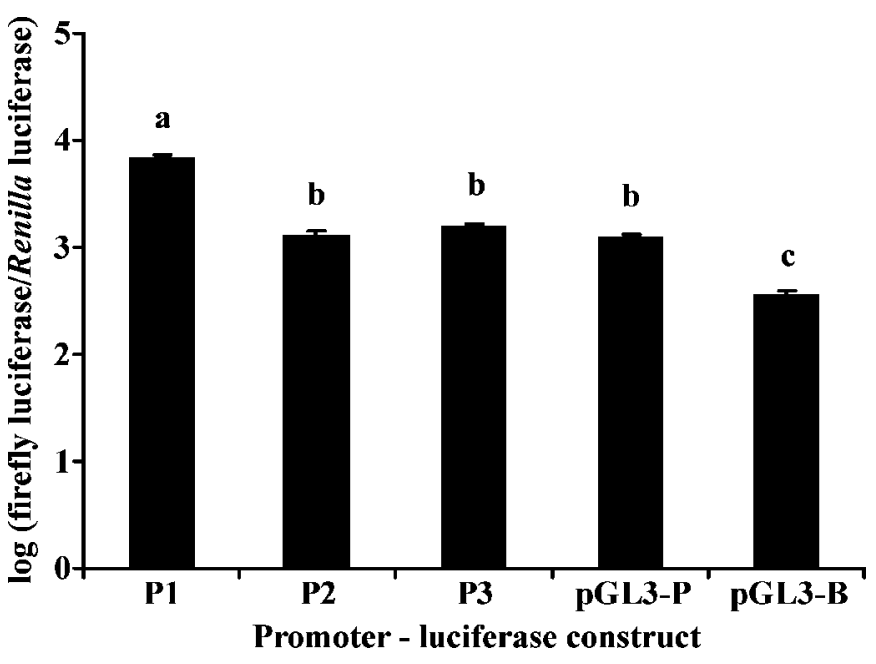

Figure 5. Luciferase activity in H4IIE cells containing bovine pyruvate carboxylase (PC) promoter - luciferase constructs. Bovine $\mathrm{PC}$ promoter constructs containing putative promoter regions of the bovine gene or pGL3-Promoter or pGL3-Basic were transfected into H4IIE cells. The plasmid pRL-CMV, which expresses Renilla luciferase, was cotransfected as a normalization control. The plasmid pGL3Basic is a promoterless luciferase plasmid and served as a negative control. The plasmid pGL3-Promoter (Promega, Madison, WI) contains a luciferase reporter driven by the SV40 promoter and served as a positive control. Luciferase expression was measured $28 \mathrm{~h}$ after transfection in 3 independent experiments. Data are least square means and standard errors of the log transformation of luciferase activity normalized to expression of a Renilla luciferase vector. All bovine PC promoters display greater luciferase activity than the promoterless luciferase plasmid (pGL3-B) $(P<0.01){ }^{\mathrm{a}-\mathrm{c}}$ Columns with different letters are different $(P<0.01)$. $P 1$ supported greater luciferase activity than $\mathrm{P} 2$ or $\mathrm{P} 3 . \mathrm{P} 1=$ promoter $1 ; \mathrm{P} 2=$ promoter $2 ; \mathrm{P} 3=$ promoter 3; pGL3-P = pGL3-promoter plasmid; and pGL3-B = pGL3basic plasmid.

moter regions between bovine and rat, mouse, or human. The presence of 3 promoters is a feature unique to the bovine PC gene that is not found in other species. Although the reasons for this additional promoter are not yet apparent, one possible hypothesis is that alternative promoters evolve to enhance recruitment of transcriptional machinery to a particular DNA site (Landry et al., 2003). In addition, the short interspersed nucleotide elements in the bovine PC gene suggest duplication and insertion of DNA sequences within the promoter regions of bovine $\mathrm{PC}$, which would explain the greater length of the bovine PC promoter compared with rat and human. Similar size differences in gene promoter regions of key genes in metabolism between domestic livestock and human, rat, or mouse orthologs have been attributed to short interspersed nucleotide element insertion (Van der Leij et al., 2002).

The exon arrangement and promoter regions of bovine $\mathrm{PC}$ generated from this work have allowed us to correlate each promoter with the PC 5' UTR variants and alternative splicing mechanisms used (Figure 1).
Accordingly, $\mathrm{P} 1$ is responsible for the transcription of PC 5' UTR A, PC 5' UTR B, PC 5' UTR C, and PC 5' UTR F. Promoter 2 is responsible for transcription of PC 5' UTR E, and P3 is responsible for transcription of PC 5' UTR D. These data provide a more precise determination of the origins of bovine PC $5^{\prime}$ UTR variants than a model proposed previously (Agca et al., 2004). Genomic sequence data reveals that the 89-bp region of bovine $\mathrm{PC} 5^{\prime} \mathrm{D}$ is formed by transcription of 2 distinct exons, a 48-bp segment (exon I) and a 41bp segment (exon II). Furthermore the 110- and 68-bp regions of $\mathrm{PC} 5^{\prime} \mathrm{C}$ and $\mathrm{F}$ are found as a contiguous 178bp segment of exon III, with an alternative transcription start site after the first $110 \mathrm{bp}$. These genomic sequence data are critical to determine the physiological mechanisms that generate variant forms of bovine PC mRNA.

Our current model places the promoter regions upstream of the exon I, II, and III. When linked to a luciferase reporter, each of these putative promoter regions was capable of driving luciferase expression in liver cells indicating that they are biologically active. The P1 construct exhibited greater luciferase activity than the P2 or the P3 construct. However, it is important to note that our analysis involved a comparison of promoter regions, not core promoter elements, and to determine the regulatory and core promoter elements within the promoter regions, further analysis is necessary. Progressive deletions of the $5^{\prime}$ end of the distal and proximal rat promoter revealed that the promoters contained both inhibitory and stimulatory elements (Jitrapakdee et al., 1997). Similar in vivo studies are necessary to ascertain the location of the regulatory regions and core promoter elements for bovine PC.

Similar to the pattern observed for rat PC $5^{\prime}$ UTR variants (Jitrapakdee et al., 1996, 1997), bovine PC 5' UTR variants display tissue specific expression (Agca et al., 2004). Expression of bovine PC $5^{\prime}$ UTR variants determined by PCR analysis demonstrated the presence of PC 5' UTR B, C, D, and E in adipose tissue, kidney, liver, brain, skeletal muscle, heart, lung, and mammary gland, whereas PC 5' UTR A and F were only detected in liver, kidney, and adipose tissue (Agca et al., 2004). Similar to the rat PC gene (Jitrapakdee et al., 1996), the variants expressed in gluconeogenic and lipogenic tissues in bovine are driven by the promoter proximal to the coding sequence. Unlike the rat, the tissue specificity observed for bovine appears to require alternative splicing within the proximal promoter. Therefore, tissue specificity for expression of PC variants is not solely linked to tissue specific promoter activation.

Promoter usage may mediate expression of PC during different physiological scenarios in bovine. In rat, mul- 
tiple promoters have been implicated in physiological regulation of $\mathrm{PC}$ expression, and proximal promoter usage appears to be linked to periods of high gluconeogenesis (Jitrapakdee et al., 1998). Although not yet characterized in bovine, it is possible that a similar phenomenon governs promoter usage because there is a disproportionate increase among PC 5' UTR variants at calving (Agca and Donkin, 2002). There is greater abundance of bPC 5' A at calving (Agca and Donkin, 2002), which suggests preferential activation of promoter 1 at calving. However, direct measures of PC promoter 1 activity are necessary to confirm this possibility. The sequence data presented here is necessary to design experiments to test the relationship between PC transcript abundance and PC promoter activation. Closer examination of the expression of bovine PC $5^{\prime} \mathrm{UTR}$ variants and response to changes in nutritional, developmental, and physiological status is necessary to fully comprehend the factors controlling PC gene promoter use in bovine. Likewise, more detailed study of the hormones and nutrients that modulated PC promoters is likely to provide insight on adaptations to nutritional and physiological changes.

\section{REFERENCES}

Agca, C., C. A. Bidwell, and S. S. Donkin. 2004. Cloning of bovine pyruvate carboxylase and 5' untranslated region variants. Anim. Biotechnol. 15:47-66.

Agca, C., and S. S. Donkin. 2002. Differential expression of pyruvate carboxylase 5'UTR variants during transition to lactation. J. Dairy Sci. 85(Suppl 1):302.
Altschul, S. F., W. Gish, W. Miller, E. W. Myers, and D. J. Lipman. 1990. Basic local alignment search tool. J. Mol. Biol. 215:403-410.

Greenfield, R. B., M. J. Cecava, and S. S. Donkin. 2000. Changes in mRNA expression for gluconeogenic enzymes in liver of dairy cattle during the transition to lactation. J. Dairy Sci. 84:12281236.

Jitrapakdee, S., G. W. Booker, A. I. Cassady, and J. C. Wallace. 1997. The rat pyruvate carboxylase gene structure. J. Biol. Chem. 272:20522-20530.

Jitrapakdee, S., Q. Gong, M. J. MacDonald, and J. C. Wallace. 1998. Regulation of rat pyruvate carboxylase gene expression by alternate promoters during development, in genetically obese rats and in insulin-secreting cells. J. Biol. Chem. 273:34422-34428.

Jitrapakdee, S., M. Slawik, G. Medina-Gomez, M. Campbell, J. C. Wallace, J. K. Sethi, S. O'Rahilly, and A. J. Vidal-Puig. 2005. The peroxisome proliferator-activated receptor-regulates murine pyruvate carboxylase gene expression in vivo and in vitro. J. Biol. Chem. 280:27466-27476.

Jitrapakdee, S., A. Vidal-Puig, and J. C. Wallace. 2006. Anaplerotic roles of pyruvate carboxylase in mammalian tissues. J. Cell Mol. Life Sci. 63:843-854.

Jitrapakdee, S., M. E. Walker, and J. C. Wallace. 1996. Identification of novel alternatively spliced pyruvate carboxylase mRNAs with divergent 5 '-untranslated regions which are expressed in a tissuespecific manner. Biochem. Biophys. Res. Commun. 223:695-700.

Landry, J. R., D. L. Mager, and B. T. Wilhelm. 2003. Complex controls: The role of alternative promoters in mammalian genomes. Trends Genet. 19:640-648.

Modrek, B., and C. Lee. 2002. A genomic view of alternative splicing. Nat. Genet. 30:13-19.

Novina, C. D., and A. L. Roy. 1996. Core promoters and transcriptional control. Trends Genet. 12:351-355.

Roeder, R. G. 1996. The role of general initiation factors in transcription by RNA polymerase II. Trends Biochem. Sci. 21:327-335.

Van der Leij, F. R., K. B. Cox, V. N. Jackson, N. C. A. Huijkman, P. Bartelds, J. R. G. Kuipers, T. Dijkhuizen, P. Terpstra, P. A. Wood, V. A. Zammit, and N. T. Price. 2002. Structural and functional genomics of the CPT1B gene for muscle-type carnitine palmitoyltransferase I in mammals. J. Biol. Chem. 277:26994-27005.

Velez, J. C., and S. S. Donkin. 2005. Feed Restriction Induces Pyruvate carboxylase but not phosphoenolpyruvate carboxykinase in dairy cows. J. Dairy Sci. 88:2938-2948. 